\title{
Differential Relationships between Momentum, Magnetic Field, Orbit Length, and Revolution Frequency
}

\author{
Steve Werkema
}

The purpose of this memo is to put down, in one place, a number of commonly used accelerator formulae. I'll also comment briefly on the derivation of these relationships. Nothing in this memo is my original work. All of this was developed many years ago by the brilliant founders of the field of Accelerator Physics.

The widely used differential relationships between beam momentum $(p)$, dipole magnetic field $(B)$, orbit length $(L)$, and revolution frequency $(f)$ are given in Table 1

- Table 1: Differential Relationships between $p, B, L$, and $f$

\begin{tabular}{|c|l|l|}
\hline Number & Variables & Relations \\
\hline \hline $\mathbf{1}$ & $p, f$, and $L$ & $\frac{d p}{p}=\gamma^{2} \frac{d f}{f}+\gamma^{2} \frac{d L}{L}$ \\
\hline $\mathbf{2}$ & $p, L$, and $B$ & $\frac{d p}{p}=\gamma_{t}^{2} \frac{d L}{L}+\frac{d B}{B}$ \\
\hline $\mathbf{3}$ & $B, f$, and $p$ & $\frac{d B}{B}=\gamma_{t}^{2} \frac{d f}{f}+\frac{\gamma^{2}-\gamma_{t}^{2}}{\gamma^{2}} \frac{d p}{p}$ \\
\hline $\mathbf{4}$ & $B, f$, and $L$ & $\frac{d B}{B}=\gamma^{2} \frac{d f}{f}+\left(\gamma^{2}-\gamma_{t}^{2}\right) \frac{d L}{L}$ \\
\hline
\end{tabular}

\section{Where do these relationships come from?}

The relationships in Table 1 are derived from three basic formulae:

(1) The formula giving beam velocity $(c \beta)$ in terms of orbit length $(L)$ and revolution frequency $(f)$ :

$$
c \beta=L f
$$

(2) The relationship between particle momentum, magnetic field, and the radius of curvature $(\rho)$ of the particle trajectory in a dipole field:

$$
\frac{p}{e}=B \rho
$$


(3) The definition of $\gamma_{t}$ (transition $\gamma$ ):

$$
\frac{d L}{L}=\frac{1}{\gamma_{t}^{2}} \frac{d p}{p}
$$

This definition is only valid for constant field (i.e. $d B=0$ ).

\section{Relationship Number 1}

The differential of equation (1) is:

$$
\frac{d \beta}{\beta}=\frac{d L}{L}+\frac{d f}{f}
$$

The beam momentum and its differential are related to $\beta$ and $d \beta$ as follows:

$$
\begin{aligned}
& p=\gamma \beta m c \\
& \left(\frac{p}{m c}\right)^{2}=\frac{\beta^{2}}{1-\beta^{2}} \\
& \frac{d p}{p}=\gamma^{2} \frac{d \beta}{\beta}
\end{aligned}
$$

Substituting $d \beta / \beta$ from equation (5) into equation (4) gives:

$$
\frac{1}{\gamma^{2}} \frac{d p}{p}=\frac{d L}{L}+\frac{d f}{f}
$$

This is relationship number 1 in Table 1. It turns out to be nothing more exciting than simple kinematics.

\section{Relationship Number 2}

The second formula in Table 1 follows from equations (2) and (3) Taking differentials of both sides of (2) gives:

$$
\frac{d \rho}{\rho}=\frac{d p}{p}-\frac{d B}{B}
$$

Differential relationship 2 in Table 1 is obtained after one determines the relationship between $d \rho / \rho$ and $d L / L$. It is clear that $d \rho / \rho$ and $d L / L$ are proportional to one another the question is: 'What is the constant of proportionality?' Temporarily calling the constant of proportionality $K$, equation (7) becomes:

$$
\begin{aligned}
K \frac{d L}{L} & =\frac{d \rho}{\rho} \\
& =\frac{d p}{p}-\frac{d B}{B}
\end{aligned}
$$


When $\mathrm{dB} / \mathrm{B}=0$ equation (8) must agree with equation (3) Thus, $K=\gamma_{t}^{2}$ and equation (8) becomes:

$$
\gamma_{t}^{2} \frac{d L}{L}=\frac{d p}{p}-\frac{d B}{B}
$$

This is identical to the second differential relationship in Table 1

\section{Relationship Number 3}

The third relationship in Table 1 follows from the first two. Solving equations (6) and (9) for $d L / L$ gives:

$$
\begin{aligned}
& \frac{1}{\gamma^{2}} \frac{d p}{p}-\frac{d f}{f}=\frac{1}{\gamma_{t}^{2}}\left(\frac{d p}{p}-\frac{d B}{B}\right) \\
& \frac{d B}{B}=\gamma_{t}^{2} \frac{d f}{f}+\left[1-\left(\frac{\gamma_{t}}{\gamma}\right)^{2}\right] \frac{d p}{p}
\end{aligned}
$$

\section{Relationship Number 4}

The last relationship in Table 1 is similarly obtained. Eliminating $d p / p$ in equations (6) and (9) gives:

$$
\begin{aligned}
& \gamma^{2}\left(\frac{d L}{L}+\frac{d f}{f}\right)=\gamma_{t}^{2} \frac{d L}{L}+\frac{d B}{B} \\
& \frac{d B}{B}=\gamma^{2} \frac{d f}{f}+\left(\gamma^{2}-\gamma_{t}^{2}\right) \frac{d L}{L}
\end{aligned}
$$

\section{Special Cases}

Several useful special cases of these relationships will be considered here.

\section{$B=$ Constant}

For the special case of constant field, formula number 3 in Table 1 gives:

$$
\begin{aligned}
\frac{d f}{f} & =-\frac{\gamma^{2}-\gamma_{t}^{2}}{\gamma_{t}^{2} \gamma^{2}} \frac{d p}{p} \\
& =-\frac{1}{\eta} \frac{d p}{p}
\end{aligned}
$$

$\eta$ is the so-called "slip factor", the definition of which is:

$$
\eta=\frac{1}{\gamma_{t}^{2}}-\frac{1}{\gamma^{2}}
$$


CAUTION: This definition is by no means universal. While all authors define $\eta$ as the difference of the inverse squares of $\gamma$ and $\gamma_{t}$, there is no universally accepted order in which this difference is taken. According to the sign convention of equation (13), $\eta$ is negative below transition and positive above transition.

The transverse movement of the beam, $\Delta x$, due to a change in momentum $\Delta p$, is given by:

$$
\Delta x(s)=D(s) \frac{\Delta p}{p}
$$

where $D(s)$ is the dispersion function evaluated at location $s$. Combining equations (12) and (14) gives:

$$
\Delta x(s)=-\frac{D(s)}{\eta} \frac{\Delta f}{f}
$$

Thus, if the value of $\eta$ is known, $D(s)$ can be measured at the location of the Beam Position Monitors (BPMs) from measurements of the change in beam position and the change in revolution frequency.

The fourth relationship in Table 1 shows how the variations of orbit length and revolution frequency are related to each other when the beam energy is changed at constant field. This interesting (although not terribly useful) relationship is given by:

$$
\frac{d L}{L}=-\frac{\eta}{\gamma_{t}^{2}} \frac{d f}{f}
$$

\section{$p=$ Constant}

Another relationship, which is useful for measuring $\gamma_{t}$, is derived from formula number 3 in Table 1 Setting $d p=0$ one gets:

$$
\frac{d f}{f}=\frac{1}{\gamma_{t}^{2}} \frac{d B}{B}
$$

Thus, by varying the bend field by a known amount and measuring the change in revolution frequency one can estimate the value of $\gamma_{t}$. 